\title{
A Rule-based Forecasting System Integrating combining and Single Forecast for Decision Making
}

\author{
Chun-Ling Lin \\ Institute of Electrical \\ and Control \\ Engineering \\ National Chiao Tung \\ University,Hsinchu,
}

Taiwan

\author{
Chen-Chun Lin \\ Institute of Management \\ of Technology \\ National Chiao Tung \\ University,Hsinchu, \\ Taiwan
}

\author{
Joseph Z. Shyu \\ Institute of \\ Management of \\ Technology \\ National Chiao Tung \\ University,Hsinchu, \\ Taiwan
}

\author{
Chin-Teng Lin \\ Institute of Electrical \\ and Control \\ Engineering \\ National Chiao Tung \\ University,Hsinchu, \\ Taiwan
}

\begin{abstract}
Many studies demonstrated that combining forecasts produces consistent but modest gains in accuracy. However, little researches define well the conditions under neither which combining is most effective nor how methods should be combined in each situation. In this paper, a rule-based forecasting system industry is proposed in order to compare forecast performance between combining forecasts and single forecasts, then define these conditions and to specify more effective combinations, finally suggest the best methods. Two comparative case studies for the telecommunications and TFT-LCD industry are proposed to examine the performance of the proposed system. Results from this study indicate that combining forecasts outperform single forecasts only when data set is data have various nonlinear characteristics. In this research, empirical evidence shows that rules based on causal forces improved the selection of forecasting methods, the structuring of time series, and the assessment of prediction intervals.
\end{abstract}

Keywords

Combining Forecast, Single Forecast, Accuracy, Multiple Forecast, Rule-based Forecasting.

\section{INTRODUCTION}

Many studies mentioned that combined forecasts with important contribution by among others [1-5]. Compared with combined forecasting, the single approaches, such as Delphi, Forecasting by Analogy, Growth Curves, Trend Extrapolation, Measures of Technology, Correlation Methods, Causal Models, Probabilistic Methods etc. [6-8], are inconsistency between forecasts [9-10], and easily fail to achieve the level of capability forecast. Due to each of themcanbe instead of another method whose forecasters may have overlooked.

However, none of each combining forecast is a universal model that is suitable for all situations. Because it is difficult to completely know the linear or nonlinear characteristics of the time series data in a real problem. For this reason, an important motivation to combine different models is the fundamental assumption that one cannot identify the true process exactly, but different models may play a complementary role in the approximation of the data generating process. Hence, a rule-based forecasting systemcould be suggested to define these conditions and to specify more effective combinations in this research.

\section{A RULE-BASED FORECASTING SYSTEM}

Traditional extrapolation forecasting methods have two important limitations. First, they do not incorporate existing knowledge that shows which extrapolation methods are the best in various conditions. Second, they ignore the managers' knowledge about the situation.

In computer science, rule-based systems (RBS) can provide an efficient way to store and manipulate knowledge to interpret information in a useful way. Rule-based forecasting $(\mathrm{RBF})$ is developed based on the concept of RBS. RBF is a type of expert system and can translate the forecasting expertise into a set of rules. From the combination which consists of simple extrapolation methods, the rules of RBF use the managers' domain knowledge and the characteristics of the data to produce a forecast. In addition, RBF is described in a rule-based forecasting process using judgment criteria in time-series extrapolation. Although the evidence for RBF's accuracy as a forecasting method is limited, the results are promising. Many original researches were done by previous research evidences.

The major advantage of RBF is to decide what methods to be used for various types of data. Thus, the findings can be applied to existing extrapolation programs. For example, the contrary series rule can be easily applied to other trend-based extrapolation models. RBF also provides a test bed for new or modified forecasting rules.

\subsection{Judgment Criteria}

In this paper, the mean square error (MSE) is employed for judgmentthe basic measure of accuracy. In statistics, MSE is used to measure the deviation between the actual and predicted values [11]. The smaller MSE corresponds to the better forecasting accuracy. Thus, MSE can be described by following equation:

$$
\begin{aligned}
& \operatorname{MSE}(f, x)=\frac{1}{n} \sum_{i=1}^{n}\left(f_{i}-\right. \\
& \left.x_{i}\right)^{2} \text {.. }
\end{aligned}
$$

where $\boldsymbol{f}_{\boldsymbol{i}}$ and $\boldsymbol{x}_{\boldsymbol{i}}$ denote the $i_{t h}$ forecast and $i_{t h}$ observation, respectively, and $n$ presents the number of forecast.

\subsection{System Design}

In this paper, a rule-based forecasting system is proposed. It consists of four different forecasting modules which are 
showed in Fig.1.The detail of these systems is described as follows:

(1) The data management subsystem: The data management sub system is an effective mechanism for storing, modifying, deleting, appending data. This subsystem can be managed by software called the database management system. In this paper, data management subsystem is designed to provide the two case studies with the capture and maintenance of current and historical data. Then, the data will be used as a base for forecasts;

(2) The model management subsystem: The model management subsystem can provide system's analytical capabilities and appropriate software management [12], by combining four various forecasting modules. When errors arise from faulty assumptions, bias, or mistakes in data, the errors can be reduced;

(3) The knowledge management subsystem: The knowledge management subsystem can support the operation of other subsystems or operate independently according to unequallyweighting-index database [12].In this subsystem, the function and activities will aim at gathering, storing and giving access to information on current states of past data. The function and activities will then accumulate the estimation of unequallyweighting parameters for using in the forecasting model and currently established operational procedures. These unequally-weightings parameters will be used to predict. This procedure will be helpful to overcome the defects from static evaluation of emergency plans and regular weight assignment and can furthest reduce the impact of the model flaw on evaluation results;

(4) The dialogue subsystem: The dialogue subsystemcan provide auser-friendly interface for decision makers to communicate with the forecasting system. It is responsible for the internal and external communications. A user sends requests to the system through this subsystem and the internal system sends feedback accordingly.

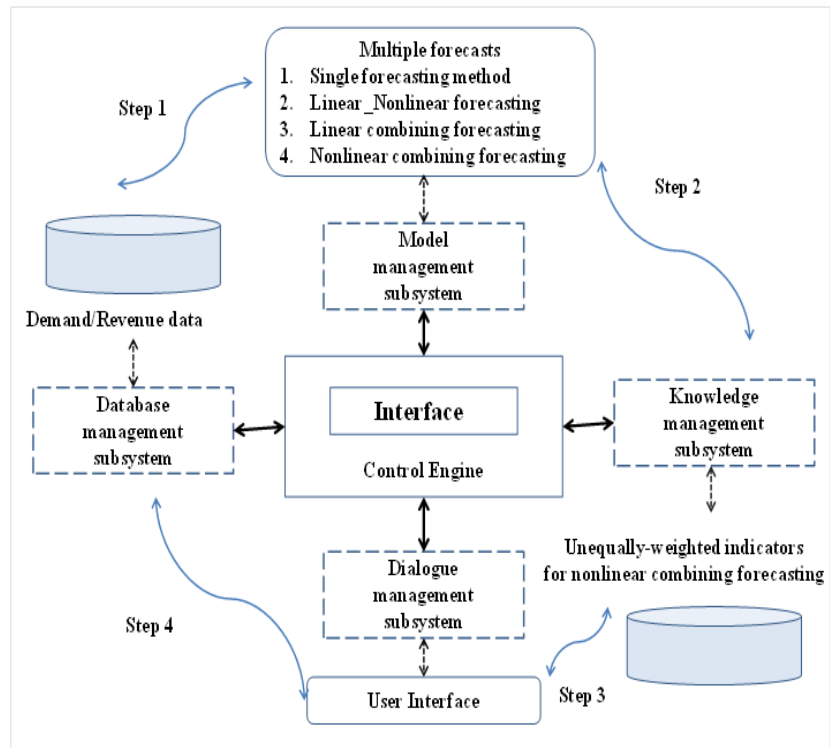

Fig.1. Rule-based forecasting system design

\section{MULTIPLE FORECASTS}

Single and combining forecasting models both have achieved successes in their own linear or nonlinear problems.In order to develop a better forecasting model, a multiple forecasts, which collecttheadvantagesform each combining method,is adopted toconstructthe rule-based forecasting system.It can be used to select better forecasting modules for better forecasting improvement.

Further, in order to tackle various forecasting problems (linear or nonlinear data characteristics) and improve forecasting accuracy, the process of the multiple forecastsis proposed in this sectionwhich isshowedin Fig. 2.This system developsfour different forecasting modules.Totally, there are 14 forecasting methods provided in those fourdifferent forecasting modules.

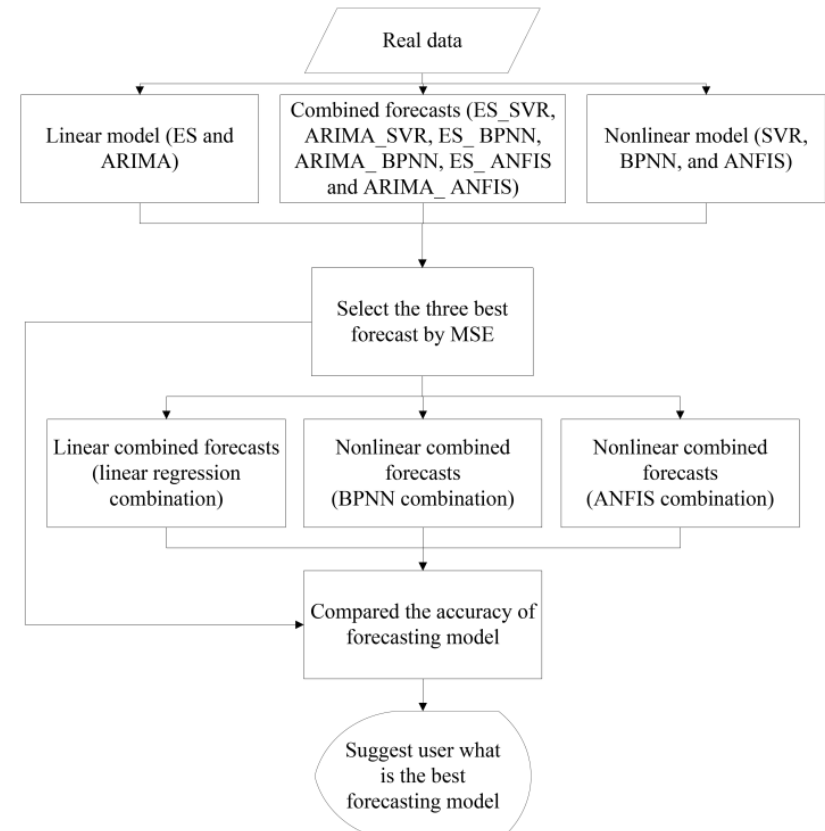

Fig.2. The process of multiple forecasts

The first moduleconsists oftwo linear and three nonlinear forecasting methods: (1)The twolinear methods are autoregressive integrated moving average model (ARIMA) and exponential smoothing model (ES); (2) The three Nonlinear methods arepropagation neural network (BPNN), adaptive neuro-fuzzy inference system (ANFIS) and support vector regression (SVR).

The second module isdeveloped by combininglinear forecasting methodsand nonlinearforecasting methods. The data (linear) willbe first forecasted by linear forecasting methods, andthe rest data (non-linear)willthenbe forecasted by non-linear forecasting methods.This module can solve an approximation of a nonlinear objective function with linear constraints using a series of linear programs [18]. There are 6 models as follow: ES_BPNN, ARIMA_BPNN, ES_SVR, ARIMA_SVR, ES_ANFIS, and ARIMA_ANFIS.

The third module is constructed bylinear combining forecasting methods. The linear regression combining forecast is also involved in this module.In the proposed method, weassume that the linear regression combining forecast have three inputs. These inputsof the linear regression combining forecastare selectedfrom the forecasts of mentionedforecasting methodsaccording their MSE. For each forecastof the forecasting methods, the linear regression combining forecastare typicallyassigned with the same weighted at each 
time frame, regardless of either global or local accuracy [13$15]$.

The final moduleis belonged to the nonlinear combining forecasting module. The final module includes two combiningforecasts:ANFIS combiningforecast and BPNN combining forecast [16-17]. Wealsoassume that there are three inputs for thetwo combiningforecasts. These inputsof the combiningforecastsare selected from the forecasts of mentioned forecasting methods according their MSE.For eachforecast offorecasting methods, different weights are applied to bothnonlinear combining forecasting methodsat each time frame.

\section{CASE STUDY}

\subsection{Case 1: Demand Forecasting of the Telecommunication Industry}

In the first case, the subject of this research mainly focuses on forecasting the future demand on the telecommunication in Taiwan, to prove the efficiency of the proposed system, and this case majorly focus on the understanding of likely market trends. The original data (from January 2006-June 2008) is from Telecommunications Carriers Association subscriber database in Taiwan in 2008 [19].

Results show thatdata characteristic in case 1 are linear as Fig. 3B. The findings also show thatSVR and ARIMA-ANFIS outperform other listing methods numerically as Fig.3A. It can be explained by that employing useful SVR(single nonlinear methods) is highly efficient to forecast linear data. Besides, we argue that the single nonlinear method can efficiently reduce forecasting error than other combining forecasting methods when analyzing linear data.

\subsection{Case2: Revenue Forecasting of theGlobal TFT-LCD Industry}

The case 2 is revenue forecasting for the TFT-LCD industry. In the last five years, TFT-LCD industry has witnessed drastic changes in the intensity of competition. The industry is going through turbulent transformation, and stepping in to the mature stage of an industry life cycle. Most companies are actually relooking at the revenues and desperate for growth. At the moment, forecasting with high accuracy is an important aid in effective and efficient planning. This paper attempts to analyze various regions fluctuating revenue data to provide a basic strategy for future demand. The monthly revenue data from October, 2009 to February, 2011 are obtained from Asia Pacific, China and North American regions.

From the experimental results listed in Table 1, we can find the effectiveness of multiple forecasts. The smaller MSE corresponds to the better forecasting accuracy. Summary results can be seen that revenue time series fluctuated in Asia pacific, China and North American regions, data are all with fluctuating nonlinearcharacteristics and forecasting results are shown in Fig. 3D, Fig. 3F and Fig.3H. The forecast performances of multiple forecasts are shown in Fig. 3C, Fig.3E and Fig.3G. The prediction results including Case1 and Case 2 are summarized as Table1 to present that which methods are higher MSE performance on forecasting. Again, according to the results in Table1, it is clear that no each model is appropriate for all situations. The assumption reason for this is that various data characteristics from different regions that also can be seen as Table1. It also implicates that the data characteristics and corresponding metrics are mapped to forecasting performance evaluation results to construct rules for forecasting method selection. To take advantage of each method, we introduce a rule-based forecasting system that can be used to select the best method.

Table 1.Summaryof Results

\begin{tabular}{|c|c|c|}
\hline Region & $\begin{array}{c}\text { Data } \\
\text { characteristics }\end{array}$ & $\begin{array}{l}\text { The best MSE forecast } \\
\text { performances and method }\end{array}$ \\
\hline \multicolumn{3}{|c|}{ Case 1: Telecommunication Industry } \\
\hline Taiwan & Linear & $\mathrm{MSE}=1.98 \mathrm{E}+11$, by SVR. \\
\hline \multicolumn{3}{|c|}{ Case 2: TFT-LCD Industry } \\
\hline $\begin{array}{l}\text { AsiaPacif } \\
\text { ic }\end{array}$ & Non-linear & $\begin{array}{c}\mathrm{MSE}=7.17 \mathrm{E}+09, \\
\text { by ES_SVR }\end{array}$ \\
\hline China & Non-linear & $\begin{array}{l}\text { MSE=7.89E+11, } \\
\text { by ANFIS non-linear } \\
\text { combination. }\end{array}$ \\
\hline $\begin{array}{l}\text { North } \\
\text { American }\end{array}$ & Non-linear & $\begin{array}{l}\mathrm{MSE}=5.44 \mathrm{E}+10, \\
\text { by SVR. }\end{array}$ \\
\hline
\end{tabular}

\subsection{System Interface}

This system has developed a procedure to optimize the choice ofrule-based forecasting system. The initial screen for the system isgiven in Fig. 4A. Once the dataset is chosen as Fig. $4 \mathrm{~B}$, the system automatically attempts to enter the historical data time, and a new screen allows the user to choosethe forecasting mode to develop acustom forecasting model. Then the system will decide which method is the best method to forecast data by the MSE performance as Fig.4C.The best method to forecast future revenue is the ES_SVR method shown in Fig.4C, and its MSE performance is 7.17E+09.

\section{CONCLUSIONS}

For previous studies, the dominant paradigm for forecasting research has been statistical modeling. These efforts have done little to incorporate domain knowledge into extrapolations.In order to bridge the research gap, a useful technical rule for forecasting is supported for variants dataanalysis and is proposed in this paper. As expected, the proposed rule-based forecasting system does better than single and combining forecasts in linear or non-linear data

For forecasting researches, the proposed system has the contributions to identify the time series problems faster, to make better use of different forecasting methods for analyzing different data, and to choose the best method with high forecast performance that can lead to better decisions and better management of corporate assets. Moreover, theautomating feature detection of the rule-based forecasting system has also introduced consistency and reliability into the forecasting process. The addedreliability could contribute to further effort in the validation and refinement of rule-based forecasting. These results are encouraging considering the absence of domain knowledge about the series in thecompetition.Becausethese findings resulted from the application of theory and empirical testing, we are optimistic that continuedrefinement of this research program will produce further improvements. 
A

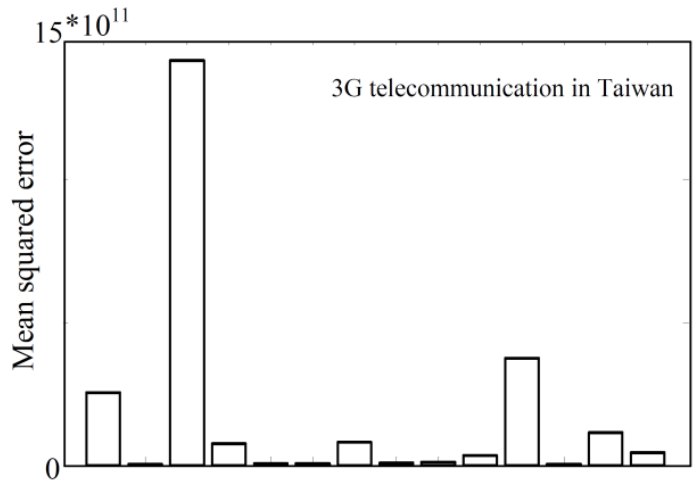

C
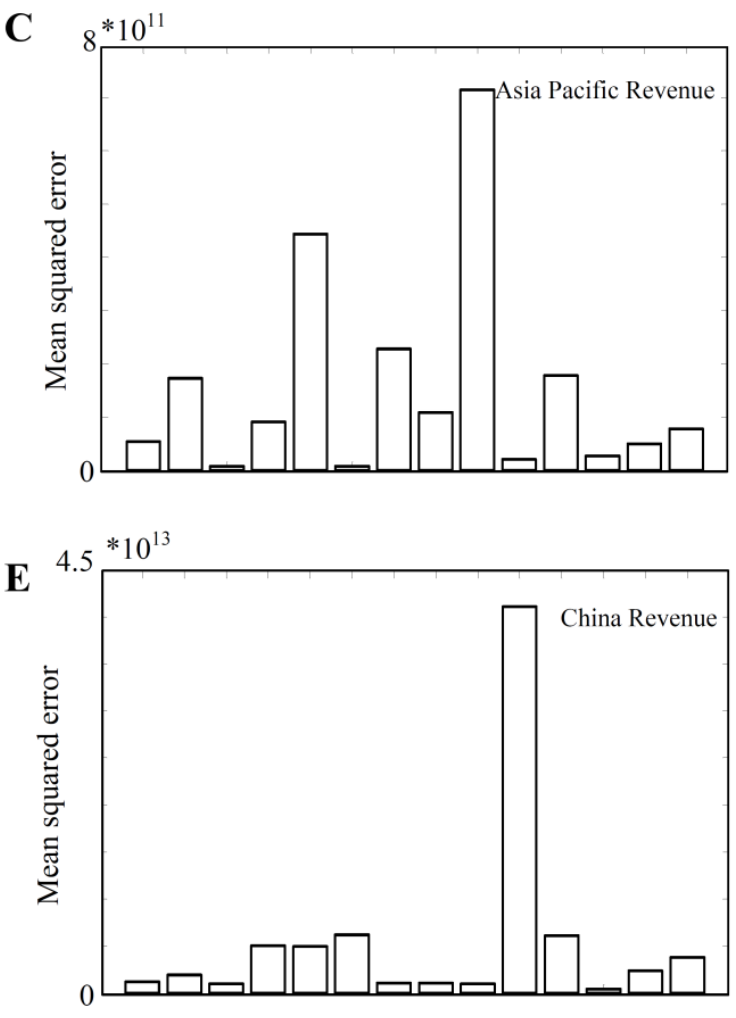

G

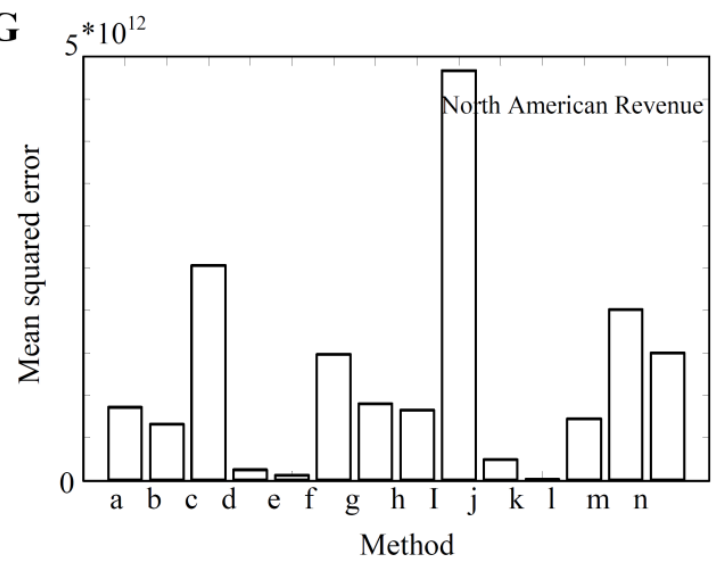

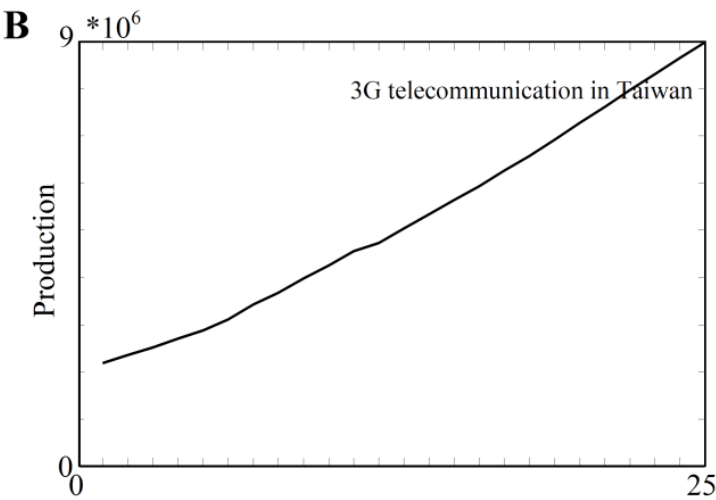
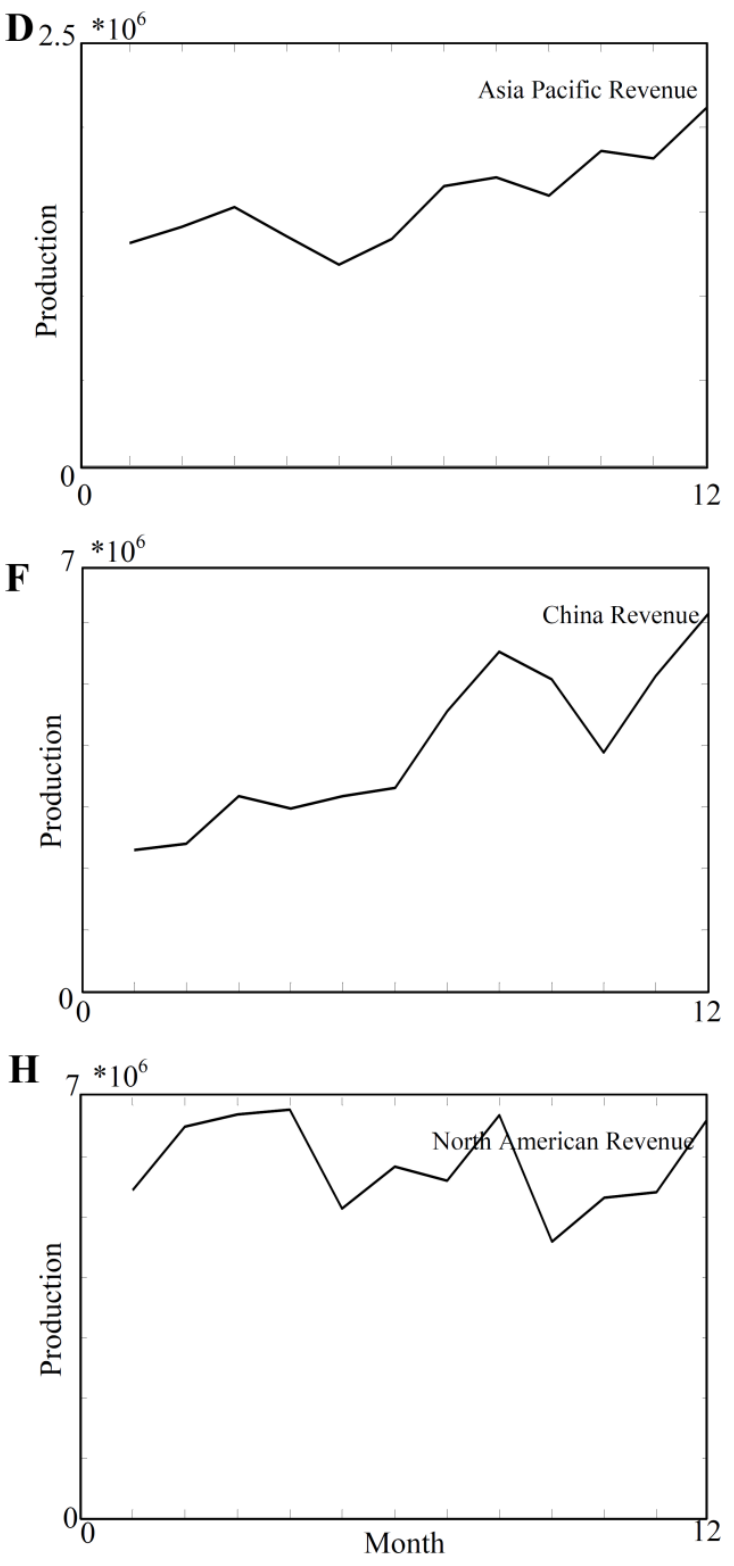

Fig 3: The performances of multiple forecasts and forecasting results

Forecasting method codes:"a" method =ES, "b" method=ARIMA; "c" method=SVR; "d" method=BPNN; "e" method=ANFIS; "f" method=ES_SVR; "g" method=ARIMA_SVR; "h" method=ES_BPNN; "i” method=ARIMA_BPNN; "j" method=ES_ANFIS; "k" method=ARIMA_ANFIS; "l" method=Linear combined forecasting; "m" method= Nonlinear combined forecasting by BPNN; " $n$ " method= Nonlinear combined forecasting by ANFIS 


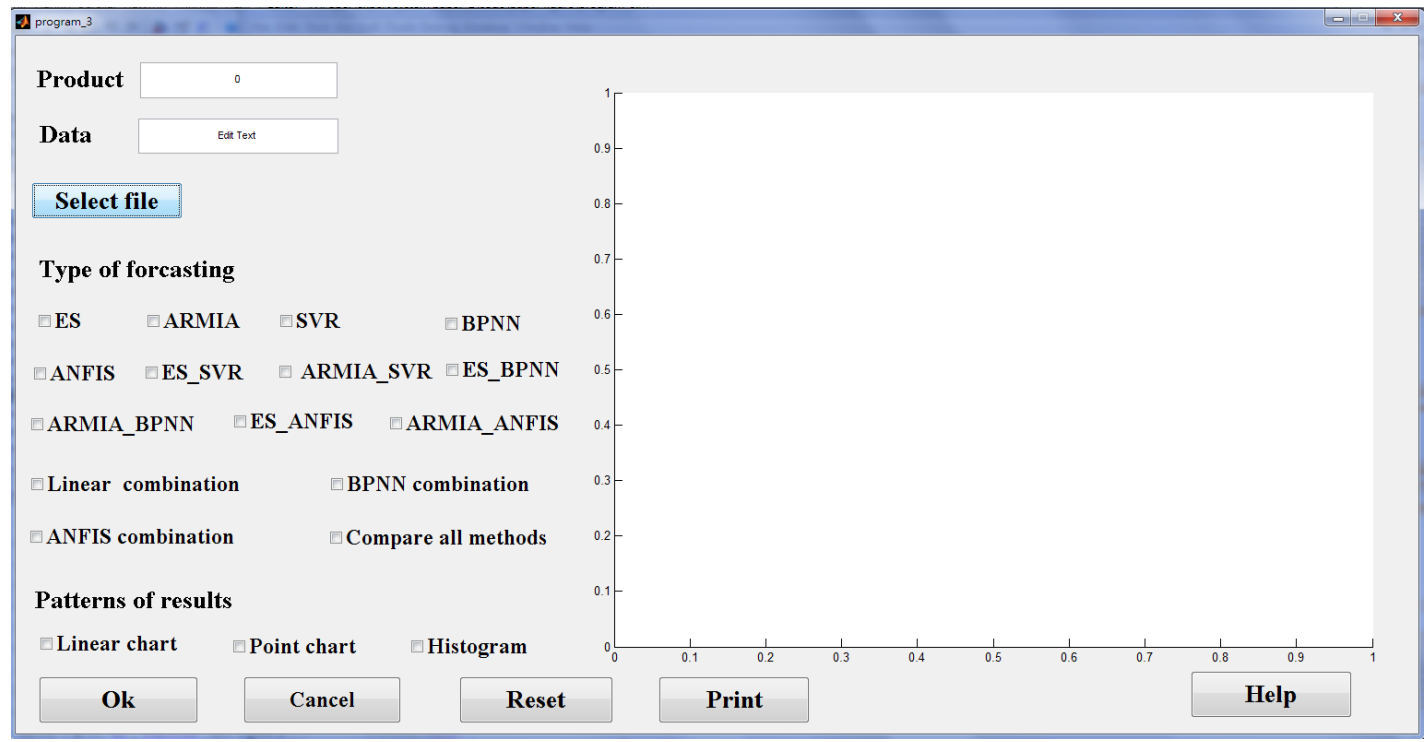

Fig.4A. System Interface (1)

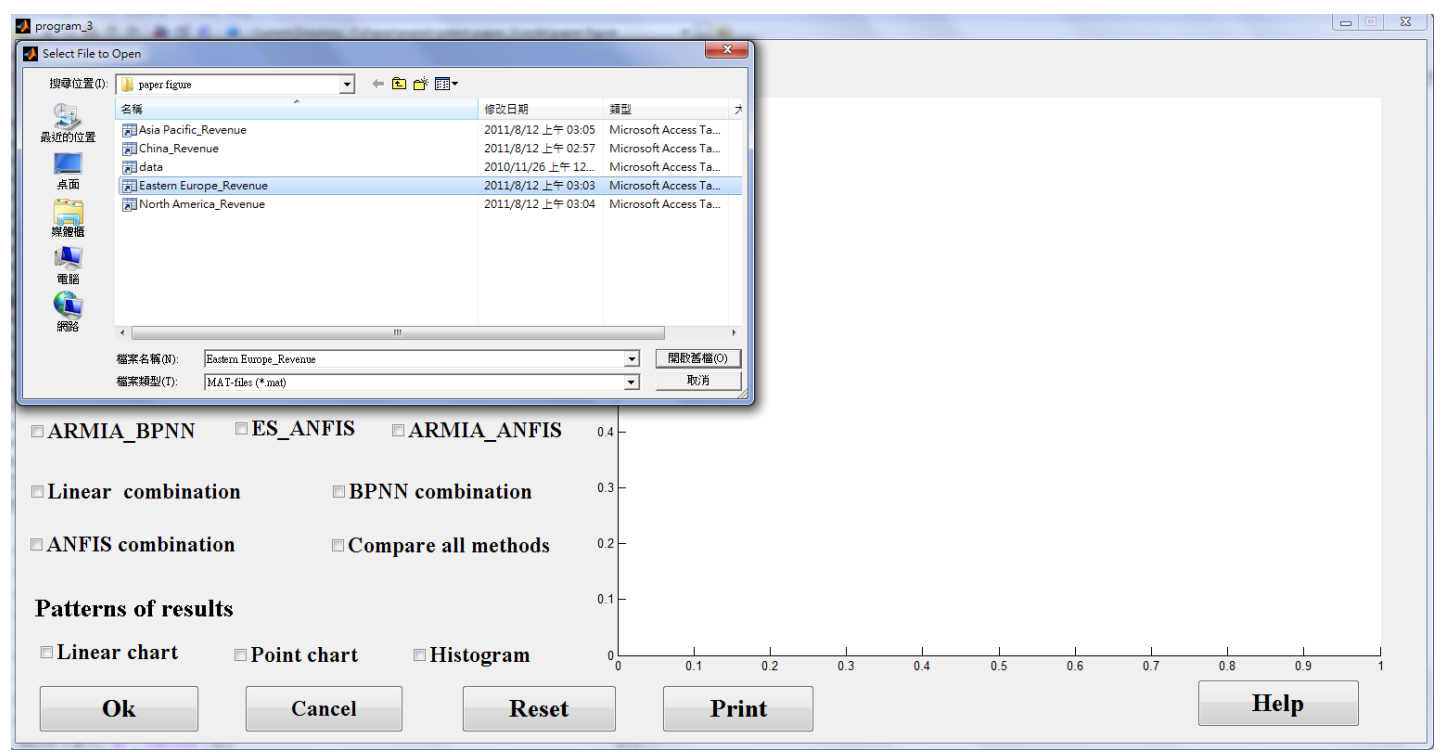

Fig.4B. System Interface (2)

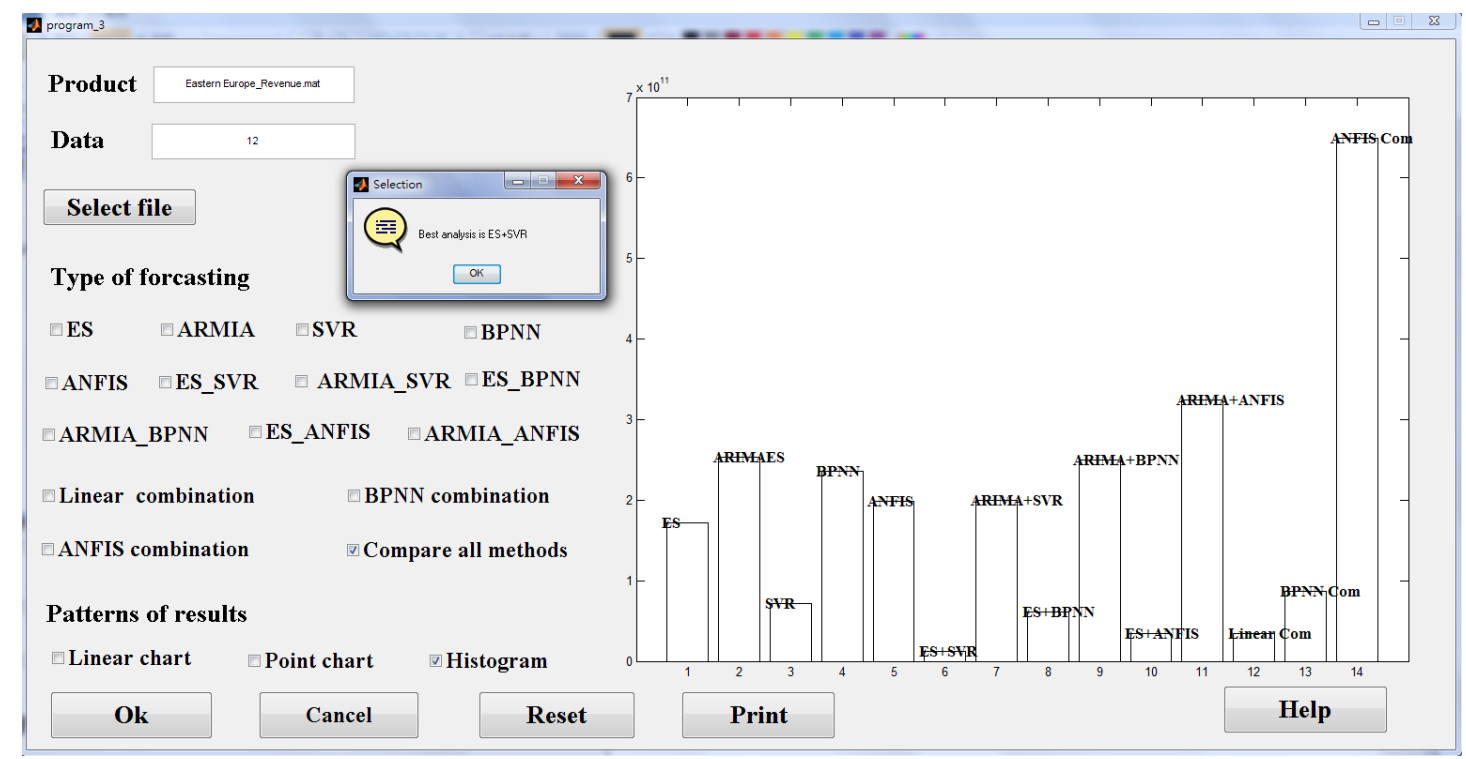

Fig.4C. System Interface (3) 


\section{ACKNOWLEDGMENTS}

We thanks for the experts who have contributed towards development of the template. This work was supported in part by the UST-UCSD International Center of Excellence in Advanced Bioengineering, in part by the Taiwan National Science Council I-RiCE Program under Grant Number NSC100-2911-I-009-101, in part by the Aiming for the Top University Plan of National Chiao Tung University, the Ministry of Education, Taiwan, under Grant Number 100W9633, also supported NSC 100-2220-E-009 060(100N113).

\section{REFERENCES}

[1] P. Poncela, J. Rodríguez, R. Sánchez-Mangas, E. Senra, Forecast combination through dimension reduction techniques, International Journal of Forecasting 26 (3) (2010) 445-626.

[2] A.S. Jore, J. Mitchell, S.P. Vahey, Combining forecast densities from VARs with uncertain instabilities, Journal of Applied economics 25(4) (2010) 621-634.

[3] J. Smith, K.F. Wallis, A Simple explanation of the forecast combination puzzle, Oxford Bulletin of Economics and Statistics 71 (2009) 331-355.

[4] M.Y. Wang, W.T. Lan, Combined forecast process: Combining scenario analysis withthe technological substitution model, Technological Forecasting and Social Change 74 (3) (2007) 357-378.

[5] M. Singh, R. Singh, V. Shinde, Application of Software Packages for Monthly Stream Flow Forecasting of Kangsabati River in India, International Journal of Computer Applications 20(3) (2011) 7-14.

[6] J.P. Martino, Technological Forecasting for Decision Making (3rd ed.), McGraw-Hill, New York, 1993.

[7] V.A.Banuls, J.L. Salmeron, A scenario-based assessment model - SBAM, Technological Forecasting and Social Change 74(6) (2007), 750-762.

[8] C.C. Holt, Forecasting seasonals and trends by exponentially weighted moving averages, International Journal of Forecasting 20(1) (2004) 5-10.
[9] M.Y. Wang, W.T. Lan, Combined forecast process: Combining scenario analysis withthe technological substitution model, Technological Forecasting and Social Change 74 (3) (2007) 357-378.

[10] Saima H., J Jaafar, S.Belhaouari, T.A. Jillani, ARIMA based Interval Type-2 Fuzzy Model for Forecasting, International Journal of Computer Applications 28(3) (2011) 17-21.

[11] G.J. Lobo, R. D. Nair, Combining judgmental and statistical forecasts: An application of earnings forecasts, Decision Sciences 21(2) (1990) 446-460.

[12] P. Gottschalk, Knowledge Management in Law Enforcement: Technologies and Techniques, Idea Group Publishing, Hershey, Pa, USA, 2007.

[13] S.I. Gunter, Nonnegativity restricted least squares combinations. International Journal of Forecasting 8(1) (1992) 45-59.

[14] R.J. Vokurka, B.E. Flores, S.P. Pearce, Automatic feature identification and graphical support in rule-based forecasting: a comparison, International Journal of Forecasting, 12(4) (1996) 495-512.

[15] P.H. Franses, R. Legerstee, Combining SKU-level sales forecasts from models and experts. Expert Systems with Applications, 38(3) (2011) 2365-2370.

[16] A. Tarsauliya, S. Kant, R. Kala, R.Tiwari, A. Shukla, Analysis of Artificial Neural Network for Financial Time Series Forecasting, International Journal of Computer Applications 9(5) (2010)16-22.

[17] G.J. Lobo, R. D. Nair, Combining judgmental and statistical forecasts: An application of earnings forecasts, Decision Sciences 21(2) (1990) 446-460.

[18] K.Y. Chen, Combining linear and nonlinear model in forecasting tourism demand, Expert Systems with Applications 38(8) (2011)10368-10376.

[19] The Ministry of Transportation and Communications (MOTC) in Taiwan, http://www.motc.gov.tw, 2011. 